\title{
Cancer and constitutional Mismatch Repair Deficiency syndrome due to homozygous MSH 6 mutation in children with Café au Lait Spots and review of literature
}

\author{
Derya Özyörük ${ }^{1 \oplus}$, Emel Ünal Cabı ${ }^{2 \oplus}$, Nurdan Taçyıldız ${ }^{2 \oplus}$, Ferda Pınarlı ${ }^{3 \oplus}$, \\ Ayşe Oğuz Erdoğan ${ }^{4 \oplus}$, Şahin Hanalioğlu ${ }^{5 \oplus}$, Arzu Yazal Erdem ${ }^{1 \oplus}$, \\ Arzu Meltem Demir ${ }^{6}$
}

Departments of ${ }^{1}$ Pediatric Hematology and Oncology, ${ }^{4}$ Pathology and ${ }^{6}$ Pediatric Gastroenterology, Health Sciences University, Ankara Childrens' Hematology and Oncology Hospital, Ankara; ${ }^{2}$ Department of Pediatric Oncology, Ankara University Faculty of Medicine, ${ }^{3}$ MYOGEN Genetic Diagnostic Center, Ankara; ${ }^{5}$ Department of Neurosurgery, Health Sciences University Dışkapı Yildırım Beyazıt Training and Research Hospital, Ankara, Turkey.

\begin{abstract}
Background. Constitutional mismatch repair deficiency (CMMRD) syndrome is a rare childhood cancer predisposition syndrome resulting from biallelic germline mutations of mismatch repair (MMR) genes. CMMRD syndrome is characterised by early onset malignancies in children.

Case. Here we present affected children of consanguinous parents diagnosed with CMMRD syndrome due to germline bi-allelic MSH 6 gene mutations with café au lait spots and multiple family cancers from Turkey and reported cases with CMMRD syndrome associated MSH 6 mutation in English literature. Hence, we reviewed English literature from 1990 to 2020 using Pub-Med database. Keywords used to search included constitutional mismatch repair deficiency syndrome, childhood cancer and MSH 6 gene mutation.

Conclusions. We emphasize that the inclusion of CMMRD syndrome in the differential diagnosis of a patient who presents with cafe' au lait spots and/or hypopigmented skin lesions and cancer especially when consanguinity and/or a history of cancer coexist in children.
\end{abstract}

Key words: childhood cancer, constitutional mismatch repair deficiency syndrome, MSH 6 mutation.

Constitutional mismatch repair deficiency (CMMRD) syndrome is a rare childhood cancer predisposition syndrome resulting from biallelic germline mutations of the DNA mismatch repair (MMR) genes. ${ }^{1}$ It is associated with a wide spectrum of malignancies including hematological, brain and intestinal tumors.

Derya Özyörük

dozyoruk@yahoo.com

Received 11th September 2020,

revised 26th November 2020, 11th January 2021,

1st February 2021, accepted 8th February 2021.

It was presented as a meeting abstract at the 51th Congress of the "Société Internationale d'Oncologie Pédiatrique" (SIOP) held in October 23-26, 2019, in Lyon, France. Also, it was presented as a meeting abstract in 61st American Society Hematology (ASH) Annual Meeting and Expositions held in December 7-10,2019 Orlando, FL, USA.
It also frequently mimics clinical features of neurofibromatosis type 1 (NF1). ${ }^{2-4}$ Although biallelic mutations in genes that regulate DNA mismatch repair, including MLH1, MSH2, MSH6 and PMS2 causes CMMRD syndrome, monoallelic germline mutations in one of these genes cause Lynch syndrome. ${ }^{4-6}$ Therefore, individuals with bi-allelic mutations have a dysfunctional mismatch repair system from birth, CMMRD syndrome is characterised by early onset malignancies. ${ }^{7-10}$ Hence, wreviewed English literature from 1990 to 2020 using Pub-Med database. Keywords used to search included constitutional mismatch repair deficiency syndrome, childhood cancer and MSH 6 gene mutation. Here we present affected children of consanguinous parents diagnosed with CMMRD syndrome due to germline bi- 
allelic MSH 6 gene mutations with café au lait spots and multiple family cancers from Turkey and reported cases with CMMRD syndrome associated MSH 6 mutation in English literature.

\section{Case Report}

A 8-year-old female was admitted to our clinic with diagnosis of a brain mass. In her past history; she was followed up with the diagnosis of NF-1 and familial mediterranean fever. Her parents were first-degree cousins with multiple cancer histories: one brother died from medulloblastoma and metachronous colon adenocarcinoma at 15 years of age and one sister died from brain tumor at 4 years of age. In addition, parents' uncle and aunt had colon adenocarcinoma and thyroid papillary cancer at 45 and 50 years of age, respectively. On physical examination, 8 to 10 cafe' au lait spots and hypopigmented skin lesions with irregular borders on her body were found. Other findings of neurofibromatosis type 1 including neurofibromas, Lisch nodules, tibia pseudoarthrosis, sphenoid wing dysplasia, and optic glioma were not seen. Laboratory investigation was within normal limits other than low serum Ig G2 levels. Cranial magnetic resonance imaging (MRI) showed a partially enhancing mass $(21 \times 34 \times 22 \mathrm{~mm})$ in the left cerebellar region, nonspesific subcortical white matter T2-FLAIR hyperintensities in frontal and parietal lobes. Also, the focal areas of hyperintense signal intensity in bazal ganglia, thalamus, mesencephalon and venous anomaly were noted. She underwent near-total resection of cerebellar mass and histopathology revealed classic desmoplastic medulloblastoma. The post-operative craniospinal MRI showed a left cerebellar hyperintensity $(12 \times 21 \mathrm{~mm})$ and enhancing lesion $(4 \times 8 \mathrm{~mm})$ without nodularity associated with recent surgery and other nonspesific intracranial findings were the same as the previous MRI. The index case was administered craniospinal irradiation (54 Gy) with a diagnosis of Medulloblastoma (Stage M2) according to Chang staging system followed by chemotherapy consisting of CCNU, cisplatin and vincristine. Because of positive family history and café au lait spots of the index case, we proceeded with genetic analysis that disclosed a novel homozygous single base insertion mutation in exon 5 of the MSH 6 gene (c.3261dupC p. Phe10881Leufs*5) (Fig. 1). NF type I and II genes were normal.

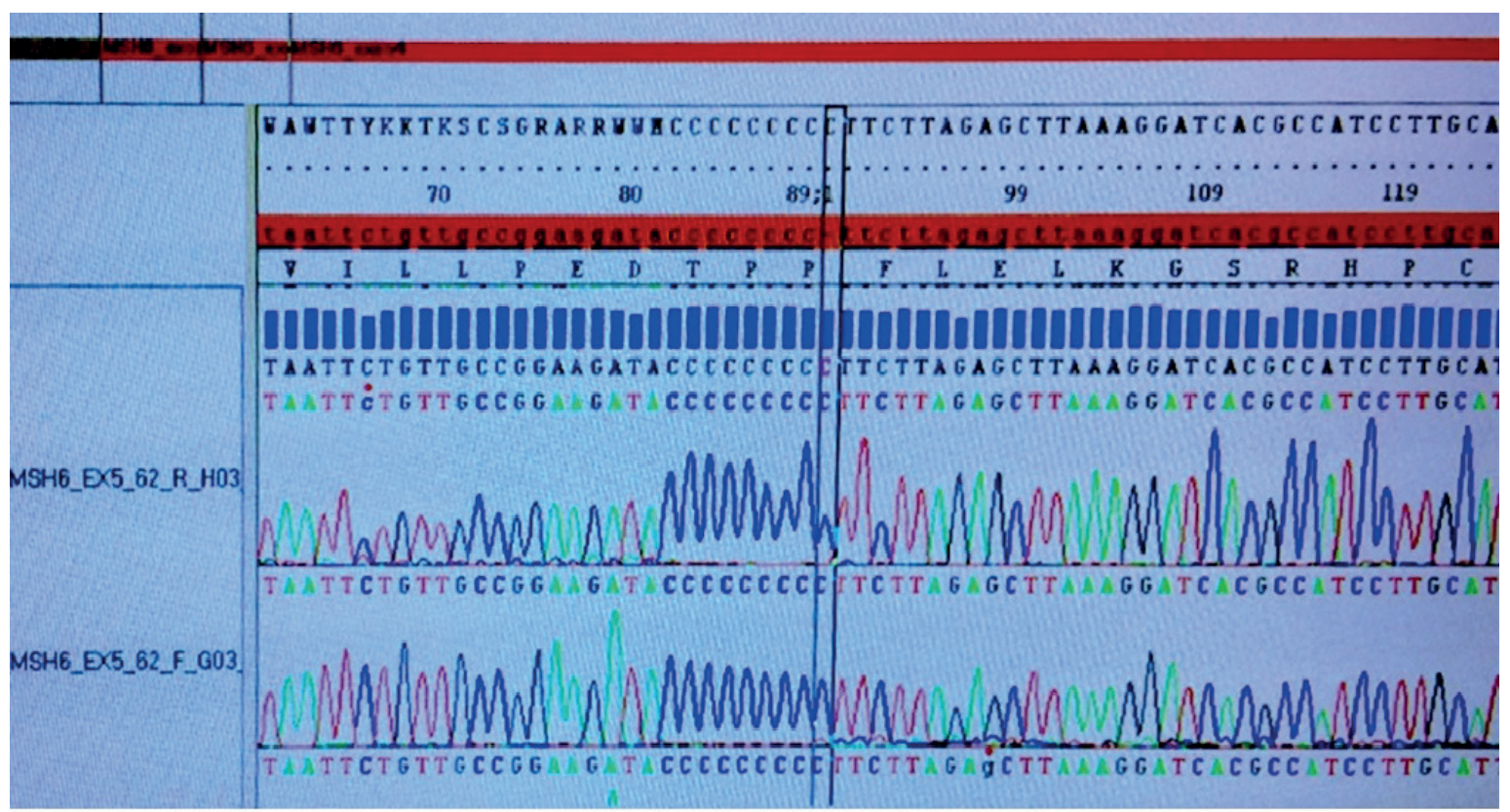

Fig. 1. MSH6 Gene Mutation of Index Case 
The genetic screening of family members were performed for MSH 6 gene mutation. The family history is shown in the pedigree in Figure 2. After 5 cycles of conventional chemotherapy, the family declined the chemotharapy due to severe hematologic toxicities and recurrent infectious complications. The colonoscopy which was normal previously revealed a new occurance of colonic tubulovillous adenomas. The chemotherapy was stopped and antiprogrammed death-1 (Anti-PD-1) drugs (nivolumab; $3 \mathrm{mg} / \mathrm{kg} / \mathrm{dose}$, every 2 weeks) were given for 24 doses. During the antiPD-1 treatment, neuroimaging findings were stable and colonic polyps were gradually decreased in number and size. One year after the end of the treatment with anti-PD-1, MRI showed a new occurance of a right cerebellar heterogenously enhancing mass (33×41x28mm) with restricted diffusion causing midline shift. Also, colonoscopy revealed new colonic polyps. She underwent tumor resection but died post-operatively. Histopathology revealed medulloblastoma. Informed consent was received from the family.

\section{Discussion}

CMMRD is considered in children with glioma, leukemia, colorectal cancer and other hereditary nonpolyposis colon cancers and any of the findings: 1) cafe' au lait spots and/ or hypopigmented skin lesions 2) history of parental consanguinity, or 3) positive family

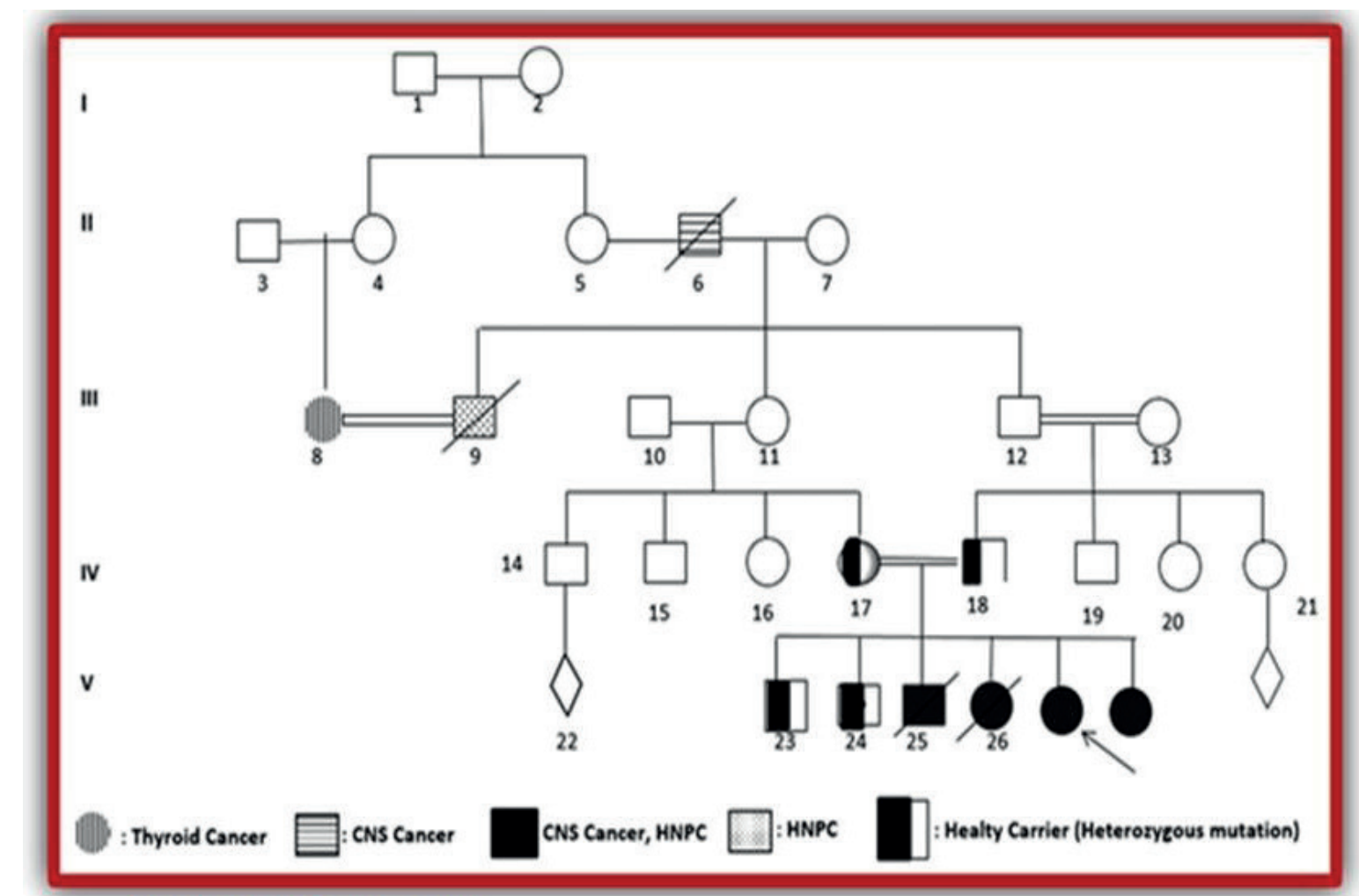

CNS: Central Nervous System

HNPC: Hereditary Non-polyposis Colorectal Cancer

Fig. 2. Five generation family tree representing cases with MSH6 mutation and/or cancers: Parents and two siblings (11 and 6years old males) are heterozygous; Sibling six-month-old is homozygous; 15-year-old deceased sibling with medulloblastoma and metastatic colorectal carcinoma was homozygous for MSH6 mutation; Other 4-year-old deceased sibling with brain tumor has no genetic test. CNS: Central Nervous System; HNPC: Hereditary Non-polyposis Colorectal Cancer 
history of hereditary nonpolyposis colon cancers. In addition, a three-point scoring system for the suspected diagnosis of CMMRD in a pediatric/young adult cancer patient has been recommended by European Consortium Tumours highly specific for CMMRD syndrome. ${ }^{9,11,12}$

The global prevalence of CMMRD is currently unknown, but rate is expected to increase in regions with a high prevalence of consanguineous marriages in especially low and middle income countries. Recently, a modified surveillance protocol for these countries with limited resources has been reported. This surveillance protocol consisted of complete blood count and fecal occult blood every 6 months, upper endoscopy/ colonoscopy and abdominal ultrasound annually, and brain MRI or CT every 6 months in children. In addition, whole body MRI was advised for surveillance as recommended in developed countries where it is available and/or reimbursed. ${ }^{13}$

According to a review of 146 cases of CMMRD syndrome conducted by Wimmer et $\mathrm{al}^{9}$, the most common mutation was PMS2 mutations (60\% of cases). Twenty-two percent and $20 \%$ of cases are caused by MLH1 or MSH2 and MSH6 mutations, respectively. ${ }^{9}$

We reviewed English literature from 1990 to 2020 using Pub-Med database. Keywords used to search included constitutional mismatch repair deficiency syndrome, childhood cancer and MSH 6 mutation. We could find approximately 38 patients diagnosed with CMMRD syndrome due to MSH 6 mutations among 20 cases and/or case series from 63 articles. The characteristics of the 38 pediatric patients with CMMRD syndrome including our patients are shown in Table I. ${ }^{1,12-31}$ The median age of onset of the first tumour was 8 years (range, 1 to 17 years). The 38 individuals with CMMRD had a total of 66 tumours (Table I). Nineteen patients had one malignancy, 13 patients had two malignancy and 7 patients had more than two malignancy. Of the 66 tumors 28 (42\%) were brain tumours, 15 (23\%) haematological malignancies, 19 (29\%) GI poliposis and/or colon cancer, and 4 (6\%) other tumours. Among the CNS tumours, the most prevalent type were glioblastoma multiforme and other high grade glial tumors (n:20; $71 \%$ ). On the other hand, we found a limited number of medulloblastoma cases (n:5;25\%) in patients with CMMRD with MSH6 gene mutations including with our patient.

The cafe' au lait spots and/or hypopigmented skin lesions mimicking NF 1 features were determined in 29 patients diagnosed with CMMRD syndrome including with our patients in Table I. The cafe' au lait spots and/ or hypopigmented skin lesions history of seven patients were not available. The cafe' au lait spots in patients with CMMRD usually differ in colour and shape from typical NF1-associated cafe au lait spots. Regardless of the genetic basis underlying clinical findings in patients with CMMRD, the majority presented cafe au lait and/or other signs indicative NF 1, although a minority fullfilled the NIH criterion for NF 1 diagnosis. ${ }^{2}$ This phenotypic overlap caused to misdiagnosis of CMMRD patients as having NF1 and impeded proper management and genetic counselling of these patients and their families similar to our patients in the past. As reported case and case series of CMMRD syndrome increase, misdiagnosis of these cases will be less in the future.

CMMRD syndrome is an autosomal recessive cancer predisposition syndrome resulting from bi-allelic mutations in MMR genes. Although consanquinity is highly suggestive for CMMRD syndrome, it can be seen in non-consanquineous families usually as compound heterozygous mutations. ${ }^{11}$ In the present review, there were consanquinity in 22 patients (58) including our patient. The consanguinity history of seven patients were not available.

Immunotherapies are directed against inhibiting receptors, such as PD-1 protein and one of its ligands programmed death-ligand 1 (PD-L1). Nivolumab, is anti-PD-1 mAb, binds PD-1 and stimulates memory response to tumor antigen-specific $\mathrm{T}$ cell proliferation. ${ }^{3}$ 


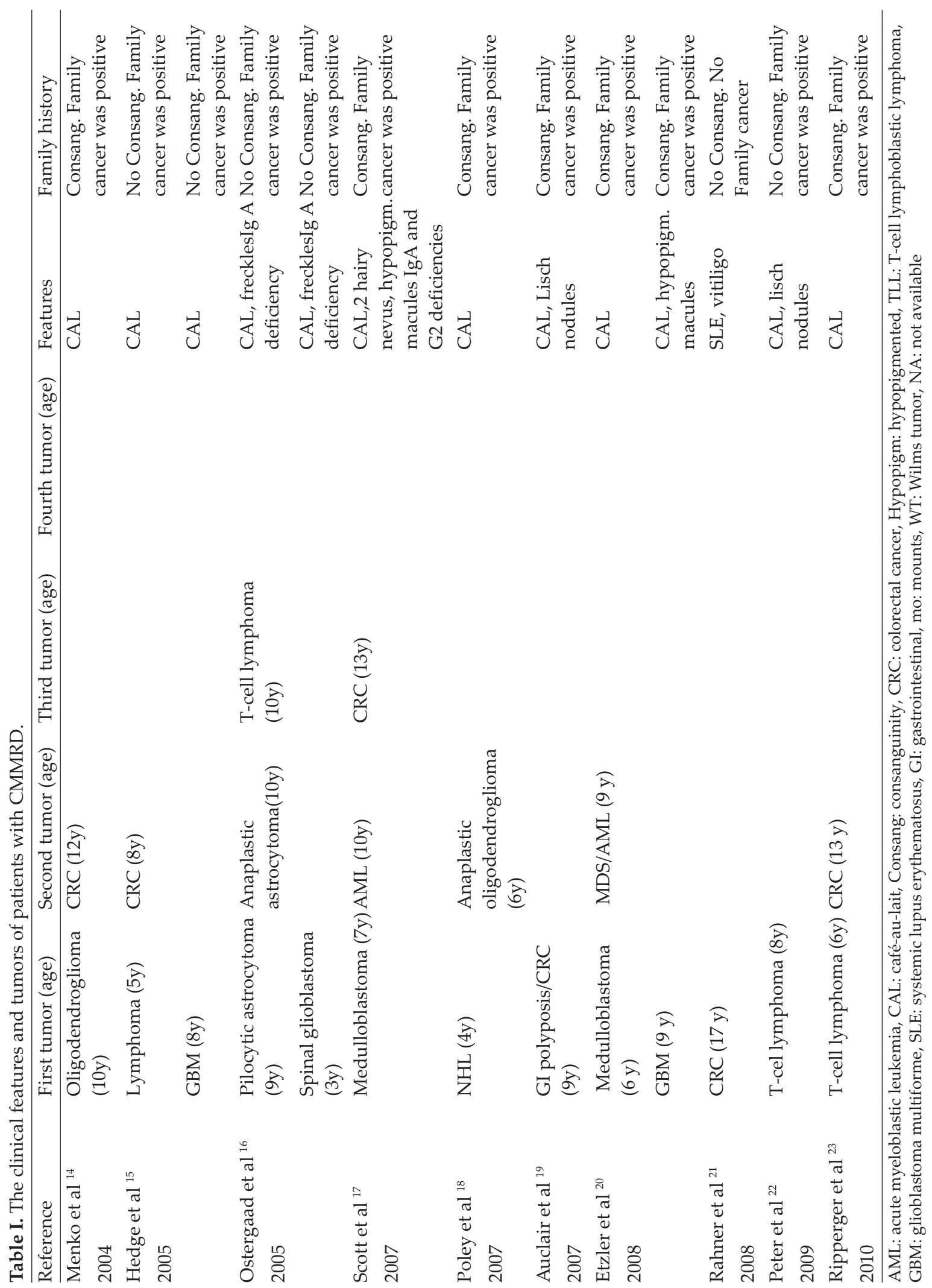




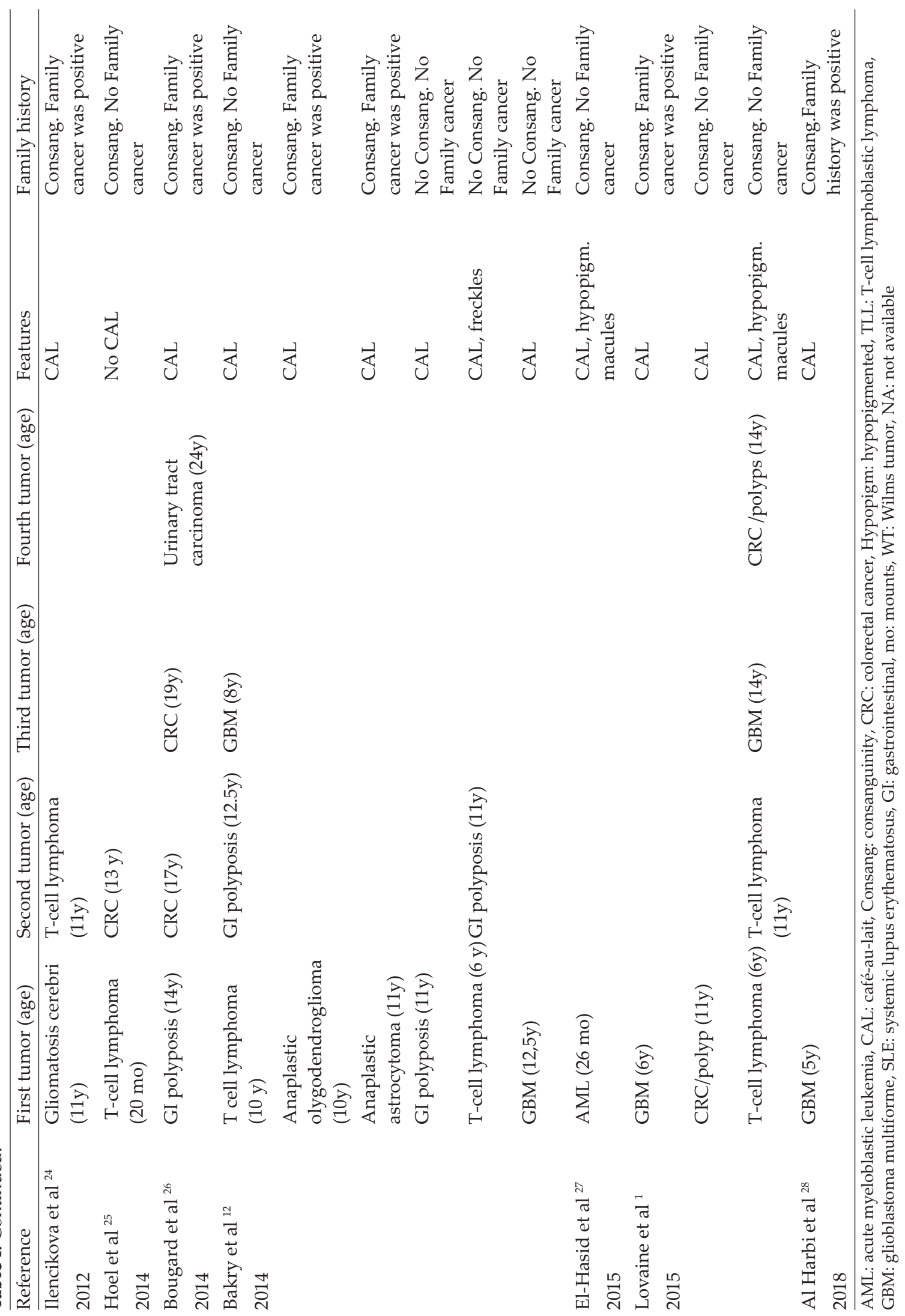




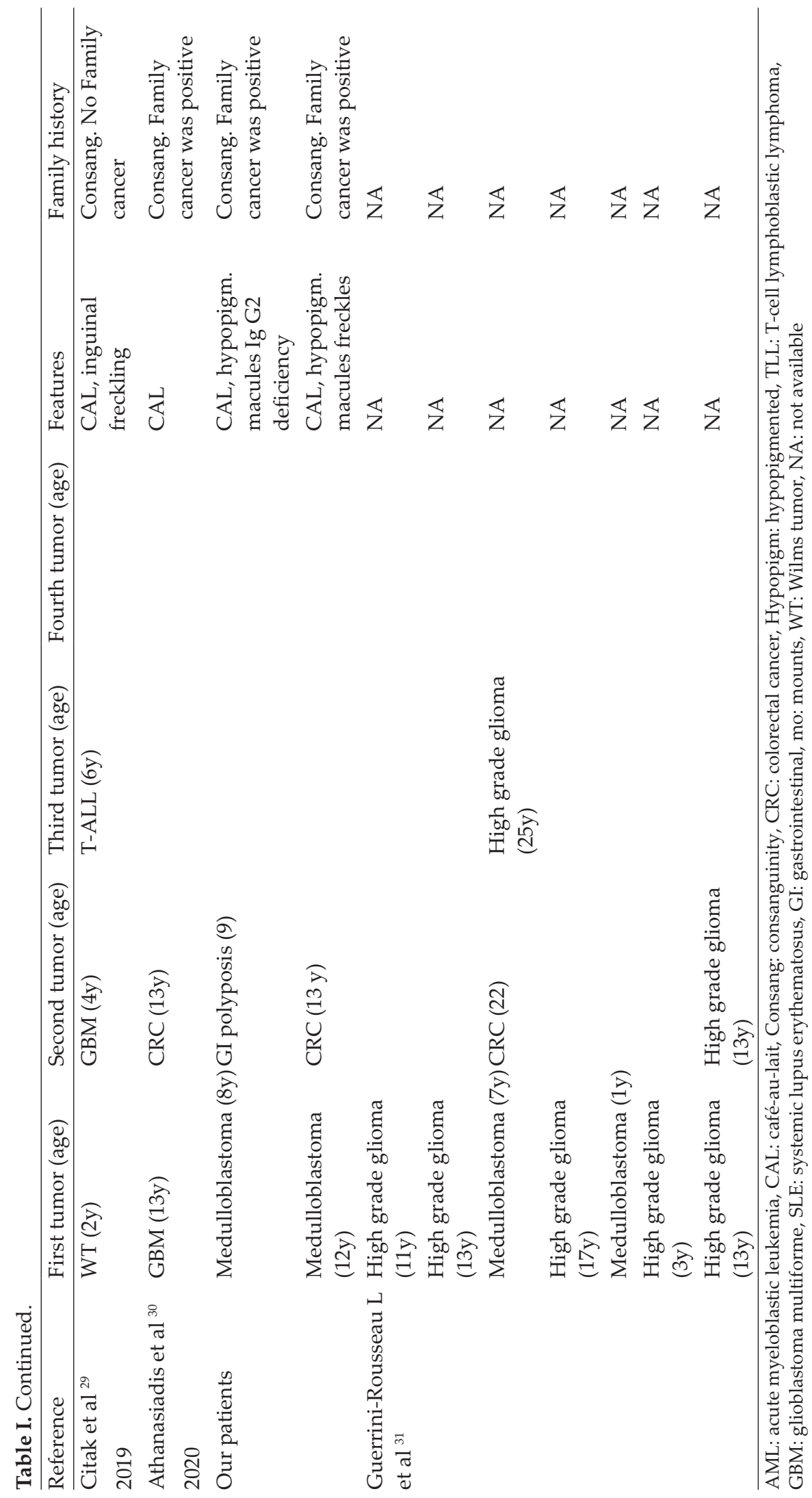


The optimal duration of immunotherapy is unknown, especially in the pediatric setting. Because the clinical findings remained stable in our index patient, nivolumab was stopped after 24 doses. Immunotherapy using immune checkpoint inhibitors have shown great promise in both adult and pediatric malignancies. First remarkable and durable responses reported were from two siblings with CMMRDassociated recurrent multifocal glioblastoma whom were treated with nivolumab (immune checkpoint inhibitor). ${ }^{6}$ After that, another study reported a 5-year-old female with CMMRD and relapsed glioblastoma multiforme whom was treated with nivolumab had durable response. ${ }^{28}$ Recently, the European C4CMMRD consortium has reported that the outcome of patients with constitutional mismatch repair deficiency (CMMRD) and brain tumor from the C4CMMRD database. According to their report, 8 patients with high grade glial tumor were administered immunotherapy with anti-PD1 antibodies at relapse. They observed disease progression in 7 of these patients within the first two months of immunotherapy and 6 of them died at 5.2 months (ranges between 1.8-9.5months) after the first injection. Therefore, they concluded that the prognosis of patients with a CMMRDrelated brain tumor (especially glioblastoma) is not as good as originally thought. ${ }^{31}$

Although significant progress has been made about cancer immunotherapy, there is no sufficient experience with prophylactic immunotherapy to prevent cancer formation especially in cancer predisposing syndrome in childhood. Because the surveillance does not quarantee detection of precancerous lesions or cancer at a curable stage, it causes a great psychological burden in families who have children with homozygous MMR gen mutations. Recently, cancer immunoprevention has been emphasized especially for healthy cases with homozygous mutation of MMR genes during surveillance to decrease the tumorogenesis. ${ }^{32,33}$

In conclusion, CMMRD syndrome is a rare and challenging disease. Because of the dismal prognosis of after cancer occurance, further studies are required to prevent cancer with immunotherapy in patients with CMMRD syndrome. In addition, we emphasize the inclusion of CMMRD syndrome in the differential diagnosis of a cancerous patient who present with cafe' au lait spots and/or hypopigmented skin lesions especially when consanguinity and/or a history of family cancer coexist in children.

\section{Author contribution}

The authors confirm contribution to the paper as follows: study conception and design: DÖ; data collection: DÖ; analysis and interpretation of results: DÖ, EUC; draft manuscript preparation: DÖ, EUC, NT, FP, EUC, NT, FP, AOE, SH, AYE, AMD. All authors reviewed the results and approved the final version of the manuscript.

\section{Conflict of interest}

The authors have no conflicts of interest to declare.

\section{REFERENCES}

1. Lavoine N, Colas C, Muleris M, et al. Constitutional mismatch repair deficiency syndrome: clinical description in a French cohort. J Med Genet 2015; 52: 770-778.

2. Wimmer K, Rosenbaum T, Messiaen L. Connections between constitutional mismatch repair deficiency syndrome and neurofibromatosis type 1 . Clin Genet 2017; 91: 507-519.

3. Westdorp H, Kolders S, Hoogerbrugge N, de Vries IJM, Jongmans MCJ, Schreibelt G. Immunotherapy holds the key to cancer treatment and prevention in constitutional mismatch repair deficiency (CMMRD) syndrome. Cancer Lett 2017; 403: 159-164.

4. Wimmer K, Etzler J. Constitutional mismatch repairdeficiency syndrome: have we so far seen only the tip of an iceberg? Hum Genet 2008; 124: 105-122.

5. Alexander TB, McGee RB, Kaye EC, et al. Metachronous T-lymphoblastic lymphoma and Burkitt lymphoma in a child with constitutional mismatch repair deficiency syndrome. Pediatr Blood Cancer 2016; 63: 1454-1456. 
6. Bouffet E, Larouche V, Campbell BB, et al. Immune checkpoint inhibition for hypermutant glioblastoma multiforme resulting from germline biallelic mismatch repair deficiency. J Clin Oncol 2016; 34: 2206-2211.

7. Ramchander NC, Ryan NA, Crosbie EJ, Evans DG. Homozygous germ-line mutation of the PMS2 mismatch repair gene: a unique case report of constitutional mismatch repair deficiency (CMMRD). BMC Med Genet 2017; 18: 40.

8. Ramachandra C, Challa VR, Shetty R. Constitutional mismatch repair deficiency syndrome: do we know it? Indian J Hum Genet 2014; 20: 192-194.

9. Wimmer K, Kratz CP, Vasen HF, et al; EU-Consortium Care for CMMRD (C4CMMRD) Diagnostic criteria for constitutional mismatch repair deficiency syndrome: suggestions of the European consortium 'care for CMMRD' (C4CMMRD). J Med Genet 2014; 51: 355-365.

10. Durno CA, Sherman PM, Aronson M, et al; International BMMRD Consortium. Phenotypic and genotypic characterisation of biallelic mismatch repair deficiency (BMMR-D) syndrome. Eur J Cancer 2015; 51: 977-983.

11. Vasen HF, Ghorbanoghli Z, Bourdeaut F, et al; EU-Consortium Care for CMMR-D (C4CMMR-D). Guidelines for surveillance of individuals with constitutional mismatch repair-deficiency proposed by the European Consortium "Care for CMMR-D" (C4CMMR-D). J Med Genet 2014; 51: 283-293.

12. Bakry D, Aronson M, Durno C, et al. Genetic and clinical determinants of constitutional mismatch repair deficiency syndrome: report from the constitutional mismatch repair deficiency consortium. Eur J Cancer 2014; 50: 987-996.

13. Kebudi R, Amayiri N, Abedalthagafi M, et al; International RRD Consortium on Low-Resource Settings Panel. Challenges and specific strategies for constitutional mismatch repair deficiency syndrome in low-resource settings. Pediatr Blood Cancer 2020; 67: e28309.

14. Menko FH, Kaspers GL, Meijer GA, Claes K, van Hagen JM, Gille JJ. A homozygous MSH6 mutation in a child with cafe-au-lait spots, oligodendroglioma and rectal cancer. Fam Cancer 2004; 3: 123-127.

15. Hegde MR, Chong B, Blazo ME, et al. A homozygous mutation in MSH6 causes Turcot syndrome. Clin Cancer Res 2005; 11: 4689-4693.

16. ØstergaardJR,SundeL,OkkelsH.Neurofibromatosis von Recklinghausen type I phenotype and early onset of cancers in siblings compound heterozygous for mutations in MSH6. Am J Med Genet A 2005: 139A: 96-105.
17. Scott RH, Mansour S, Pritchard-Jones K, Kumar D, MacSweeney F, Rahman N. Medulloblastoma, acute myelocytic leukemia and colonic carcinomas in a child with biallelic MSH6 mutations. Nat Clin Pract Oncol 2007; 4: 130-134.

18. Poley JW, Wagner A, Hoogmans MM, et al; Rotterdam Initiative on Gastrointestinal Hereditary Tumors. Biallelic germline mutations of mismatchrepair genes: a possible cause for multiple pediatric malignancies. Cancer 2007; 109: 2349-2356.

19. Auclair J, Leroux D, Desseigne F, et al. Novel biallelic mutations in MSH6 and PMS2 genes: gene conversion as a likely cause of PMS2 gene inactivation. Hum Mutat 2007; 28: 1084-1090.

20. Etzler J, Peyrl A, Zatkova A, et al. RNA-based mutation analysis identifies an unusual MSH6 splicing defect and circumvents PMS2 pseudogene interference. Hum Mutat 2008; 29: 299-305.

21. Rahner N, Höefler G, Högenauer C, et al. Compound heterozygosity for two MSH6 mutations in a patient with early onset colorectal cancer, vitiligo and systemic lupus erythematosus. Am J Med Genet A 2008; 146A: 1314-1319.

22. Peters A, Born H, Ettinger R, Levonian P, Jedele KB. Compound heterozygosity for MSH6 mutations in a pediatric lymphoma patient. J Pediatr Hematol Oncol 2009; 31: 113-115.

23. Ripperger T, Beger C, Rahner N, et al. Constitutional mismatch repair deficiency and childhood leukemia/ lymphoma--report on a novel biallelic MSH6 mutation. Haematologica 2010; 95: 841-844.

24. Ilencikova D. Constitutional mismatch repairdeficiency syndrome (CMMR-D)- a case report of a family with biallelic MSH6 mutation. Klin Onkol 2012; 25(Suppl): S34-S38.

25. Hoell JI, Gombert M, Ginzel S, et al. Constitutional mismatch repair-deficiency and whole-exome sequencing as the means of the rapid detection of the causative MSH6 defect. Klin Padiatr 2014; 226: 357-361.

26. Bougeard G, Olivier-Faivre L, Baert-Desurmont S, et al. Diversity of the clinical presentation of the MMR gene biallelic mutations. Fam Cancer 2014; 13: 131135.

27. Elhasid, R, Dvir R, Keidar R, et al; BMMRD Consortium. Management of acute myeloblastic leukemia in a child with biallelic mismatch repair deficiency. J Pediatr Hematol Oncol 2015; 37: e490-e493. 
28. AlHarbi M, Ali Mobark N, AlMubarak L, et al. Durable response to nivolumab in a pediatric patient with refractory glioblastoma and constitutional biallelic mismatch repair deficiency. Oncologist 2018; 23: 1401-1406.

29. Citak EC, Sagcan F, Gundugan BD, et al. Metachronous Wilms tumor, glioblastoma, and T-cell leukemia in an child with constitutional mismatch repair deficiency syndrome due to novel mutation in MSH6 (c.2590G>T). J Pediatr Hematol Oncol 2021; 43: e198-e202.

30. Athanasiadis DI, Athanasiadou KI, Voulgaridou A, et al. Coexistence of constitutional mismatch repair deficiency syndrome and Lynch syndrome in a family of seven: MSH6 mutation and childhood colorectal cancer - a case series. Acta Gastroenterol Belg 2020; 83: 479-481.
31. Guerrini-Rousseau L, Varlet P, Colas C, et al Constitutional mismatch repair deficiencyassociated brain tumors: report from the European C4CMMRD consortium. Neurooncol Adv 2019; 1: vdz033.

32. Lenders EKSM, Westdrop $\mathrm{H}$, Brüggeman RJ et al. Cancer prevention by aspirin in children with Constitutional Mismatch Repair Deficiency (CMMRD). Eur J Hum Genet 2018; 26: 1417-1423.

33. Unal E, Ozyoruk D, Tacyildiz N, et al. ANTI-PD-1 treatment in a family with constitutional mismatch repair deficiency syndrome with multiple cancers from Turkey. Is cancer immunoprevention with checkpoint inhibitors HAS a role in Cases with homozygous mutationis? Blood 2019; 134(Suppl 1): 5632 . 